\title{
ACUTE PULMONARY EDEMA
} AND ELEVATED TROPONIN

\author{
what is their significance? clinical review
}

\begin{abstract}
Maria das Neves Dantas da Silveira Barros*, Vander Weyden Batista de Sousa**, Isabelle Adjanine Borges de Lima***, Cecília Raquel Bezerra Marinho Nóbrega***, Isabelle Conceição Albuquerque Machado Moreira****, Suzana Marine Duarte Martins Dourado****, Bruna Maria Simões Andrade****, Virgínia da Silva Batista****, Maria Cleide Freire Clementino da Silva******

Corresponding author: Maria das Neves Dantas da Silveira Barros - mndantas@hotmail.com.br

* PhD Student in BAHIANA - School of Medicine and Public Health. Master's degree in Medicine in São Paulo University. Medical doctor at the Pernambuco Cardiovascular Emergency Room, Brazil

** Clinical Cardiology Intern at FCM/UPE and at the Pernambuco Cardiovascular Emergency Room - PROCAPE. Medical doctor at Prontolinda, Pernambuco, Brazil

*** Intern at the Pernambuco Cardiovascular Emergency Room - PROCAPE

**** Medical doctor's degree in the University of Pernambuco, Brazil

***** Biologist's degree in the University of Pernambuco, Brazil

****** Biomedic at the Permanbuco University Central Laboratory, Brazil
\end{abstract}

\begin{abstract}
Acute pulmonary edema (APE) is a clinical condition characterized by severe acute respiratory distress, frequently accompanied by crackling lung sounds and sudoresis. One classification system divides APE into cardiogenic or non-cardiogenic adult respiratory distress syndrome - ARDS. This study reviews cardiogenic APE, which is the most severe clinical presentation of heart failure (ADHF), and its relationship with the increase of troponin along with other factors such as abnormalities in the electrocardiogram (ECG) that may be mistaken for acute coronary syndrome (ACS). Atypical symptoms could occur in the presentation of ACS in $8,4 \%$ of cases, as has been shown in the Global Registry of Acute Coronary Events (GRACE): dyspnea (49\%), sudoresis (26\%), nauseas or vomiting (24\%) and syncope (19\%). The CK-MB enzyme was replaced by cardiac troponin (cTn) as the chosen marker of myocardial necrosis in the diagnosis of AMI in the late gos. The cTn is a marker of cardiac damage, not just cardiac ischemia, and this may pose questions as whether there is a myocardium infarction or not. The positive results of a cTn should be interpreted considering the clinical signs of myocardial ischemia. The challenge remains and further studies are needed to aid in accurate diagnosis of both conditions (APE and ACS) as well as cases in which one results from the other because the role of coronary artery disease in acute heart failure has not been well studied in clinical trials.

Keywords: Pulmonary Edema; Heart Failure; Acute Coronary Syndrome; Troponin.
\end{abstract}




\section{LITERATURE REVIEW}

Acute pulmonary edema (APE) is a clinical condition characterized by severe acute respiratory distress accompanied by crackling lung sounds and most often, intense sudoresis.

One of the classifications divides APE into cardiogenic and non-cardiogenic categories (adult respiratory distress syndrome - ARDS). Amongst these two types, there are pathophysiological differences. The first is caused by increased hydrostatic pressure in the pulmonary capillary bed and the second type by increased permeability in the alveolar-capillary membrane.

The difference between the two forms is not always possible to define, but the clinical history is of great value, since the diseases related to each type are well recognized. Diagnostic error may occur due to coexistence of other diseases in the same individual. The leading causes of cardiogenic APE are diseases in the left ventricle or the left atrium while noncardiogenic APE (ARDS) may be caused by infection (pneumonia, sepsis) and trauma with multiple transfusions and aspiration. Paroxysmal nocturnal dyspnea, orthopnea, presence of murmurs or third heart sound increase the possibility of cardiac etiology for the APE; however, the accuracy to make the diagnosis of these findings is low. ${ }^{(1)}$

The focus of this review is the cardiogenic APE, which is the most severe clinical presentation of heart failure (ADHF), and its relationship with the increase of troponin and other factors, such as abnormalities in the electrocardiogram (ECG) which may be mistaken for acute coronary syndrome (ACS), since atypical symptoms could occur in the presentation of ACS in $8,4 \%$ of the cases as shown in the Global Registry of Acute Coronary Events (GRACE): dyspnea is the most common presentation (49\%), followed by sudoresis $(26 \%)$, nauseas or vomiting (24\%) and syncope (19\%). ${ }^{(2)}$

The pathological process of cardiogenic APE is due to plasma extravasation within the lung interstice and alveoli, resulting from increased left ventricular filling pressure and therefore, pulmonary capillary pressure, usually at levels above $18 \mathrm{mmHg}$. The exact mechanism of the sudden increase in pulmonary capillary pressure is not clearly established yet; however, the accepted pathophysiological factor is the loss of myocardial compliance.

Decompensated heart failure is a frequent condition in the emergency rooms and a public health concern due to the increased number of hospitalizations it causes. ${ }^{(3)}$ It is one of the most frequent causes of morbidity and mortality in the industrialized world and is the leading cause of hospitalization in patients over 65 years old. $(4,5)$ Therefore, in light of the aging population, the number of cases of HF tends to increase and consequently, their hospitalization.

Acute Pulmonary Edema (APE) was considered, among the modalities of acute HF, second in hospital costs, being exceeded only by cardiogenic shock. ${ }^{(6)}$ Often, their anatomical substrate is severe coronary artery disease (CAD), ${ }^{(7)}$ and the main cause of chronic heart failure (HF). ${ }^{(8)}$

The factors involved in HF exacerbation are: interruption or non-adherence to the treatment, myocardial ischemia, infection, cardiac arrhythmias and anemia. ${ }^{(9)}$ It is considered one of the greatest life threatening cardiovascular emergencies. For this reason, the diagnosis and therapy requires quick medical attention.

In the absence of previous heart disease, the causes of APE to be considered are: fluid overload, hypertension, acute dysfunction of the mitral and/ or aortic valve, renal disease and exacerbation of a systolic or diastolic heart failure. Cardiac tumors and pulmonary embolism are other possible causes.

Another cause related to APE, often defined as a synonym for "flash" edema is the renal artery stenosis, recognized by Pickering in 1988 and designated Pickering syndrome by Messerli et al, which defined "flash edema" as the term to describe more dramatically decompensated heart failure (APE), and not necessarily renal artery stenosis. The prevalence of renal artery stenosis > 50\% is estimated at $8 \%$, ranging from 3.1 to $22.9 \%$ in patients with high probability of disease who were 
referred for investigation of renal artery stenosis. Around $20 \%$ of these patients have significant stenosis. ${ }^{(10)}$

The diagnosis of APE is essentially clinical, with sudden dyspnea, crackling in pulmonary auscultation, cold skin and sometimes intense sudoresis as symptoms. The people at risk of developing this disease are adults with diabetes mellitus (DM), hypertension and heart disease.

Chest radiography is considered by many authors as a criteria to identify the APE. Pulmonary venocapillary congestion and interstitial edema are the most specific findings. However, in $20 \%$ of acute heart failure situations there are no findings, mainly in patients with CHF. ${ }^{(1)}$ The hypoxemia that accompanies the intense respiratory distress can be detected by blood gas analysis or pulse oximetry.

Routine laboratory tests, such as blood count, urea, creatinine, potassium and glucose should be requested. Biomarkers such as troponin and B-type natriuretic peptide investigations must be ordered depending on the clinical conditions, although some authors recommend mandatory troponin dosages, justified by the association between APE and ischemic heart disease. The B-type natriuretic peptide (BNP) or its N-terminal precursor (NT-pro-BNP) have been shown to be useful in the evaluation of dyspnea, especially when the etiology is uncertain. ${ }^{(12)}$ Both have shown a better performance in the diagnosis of acute HF than chest X-ray, as it has been showed in a multivariate model in which the NTpro-BNP was the strongest predictor to acute heart failure and the interstitial edema on chest $\mathrm{X}$-ray was present only in $42 \%$. $^{(13)}$

The electrocardiogram (ECG) does not provide diagnosis, but it may suggest the cause and/or precipitating factor of HF. The findings with greater likelihood ratio (LR) for heart failure highlight the atrial fibrillation ( $L R=3.8,95 \% \mathrm{Cl} 1.7$ to 8.8), followed by new $\mathrm{T}$ waves $(\mathrm{LR}=3.0,95 \% \mathrm{Cl}$ from 1.7 to 5.3$) .^{(14)}$ Echocardiography is a very useful examination to assess ventricular function and to define the therapy after the episode. Coronary angiography should be ordered, undoubtedly, if the clinical course is related to the acute phase of acute myocardial infarction with ST-segment elevation (STEMI).
The classic treatment for this serious syndrome consists of intravenous diuretics, vasodilators - venous and sublingual - and oxygen therapy. Diuretics are drugs still classically used in the treatment of acute HF, despite the lack of evidence of their best mode of administration and effect on mortality rates. ${ }^{(15,16)}$ Because of the severity of APE, intravenous administration is the routine prescription. Non-invasive ventilation has been recommended in the treatment of this respiratory discomfort because it reduces mortality and the need for intubation. ${ }^{(17)}$

The association between APE and acute myocardial infarction (AMI) is described by several authors and in epidemiological surveys. Due to the lack of specificity of clinical, electrocardiographic and biomarkers used in the diagnosis of acute coronary syndrome (ACS), the prevalence of this association - APE and AMI - may vary and do not represent, in fact, the reality, overestimating the diagnosis of AMI associated to APE. Knowing that myocardial ischemia is one of the main factors involved in the genesis of the APE does not mean that there is an ongoing $\mathrm{AMI}$, especially if the troponin is elevated, because dyspnea is not the most frequent presentation of $\mathrm{AMI}$, neither is positive troponin pathognomonic of heart attack.

Javed et al. showed that only $34,2 \%$ of patients with increased troponin had AMI, reinforcing the importance of recognizing the causes that lead to its increase and demonstrating that $2 / 3$ of troponin are not due to heart attack. ${ }^{(18)}$

Some authors have defined APE with a positive myocardial necrosis marker (MNM) as an clinical expression of a ACS without ST elevation, $(7,19)$ ie, when the MNM are raised in the context of APE, they consider as a non-ST-elevation AMI associated $A P E$, since $C K M B$ rises twice the normal reference value with or without ECG changes. This is probably the reason that some doctors interpret that a positive necrosis marker in the APE is associated with an acute coronary event. On the other hand, the markers should not be used with diagnostic purposes in patients with STEMI and the doctors should not wait for the results to come in before starting to treat patients. ${ }^{(20)}$ 
Troponin (Tn) is a protein of the thin filament of cardiac and skeletal muscle. The troponin complex is composed of three distinct proteins, each participating in the process of regulating the excitation-contraction of the muscle fiber. This complex consisting of $\mathrm{Tnl}, \mathrm{TnT}$ and $\mathrm{TnC}$. $\mathrm{TnC}$ is expressed genetically in the skeletal muscle and $\mathrm{Tnl}$ and $\mathrm{TnT}$ are specific to the heart muscle, thus called cardiac troponin - cTn. They are usually undetectable in the circulation and when are released by the heart muscle could represent a cardiac injury signal. Any type of injury, not only ischemic, can cause the cTn to be detected in the serum. New laboratory tests have allowed greater accuracy for detecting cTn since they were first introduced in the 1990s, ${ }^{(21)}$ when they were first used as a risk marker in ACS and as a marker of necrosis in 2000, when the first universal definition of myocardial infarction was written. ${ }^{(22)}$

Since then, the CK-MB enzyme was replaced by cardiac troponin (cTn) as the marker of myocardial necrosis of choice in the diagnosis of AMI. Given that cTn is a marker of cardiac damage, not just cardiac ischemia, and also that in many cases there is a high probability of association with atherosclerotic disease, but not necessarily with acute ischemia, only clinical symptoms and subsequent evaluations may clarify the cause of increased cTn, which are different, as shown below in table - $7^{(23)}$

Table 1 - Increased Cardiac Troponin in the absence of ischemic heart disease

\section{1) Danage caused by secondary myocardial ischemia (AMI type 2)}

Tachyarrhythmia and bradyarrhythmia

Aortic dissection

Hypertension or hypotension (e.g.: hemorrhagic shock, hypertensive emergency)

Severe aortic valve disease

Acute and chronic heart failure with or without coronary heart disease

Hypertrophic cardiomyopathy

Coronary vasculitis (e.g.: systemic lupus erythematosus, Kawasaki disease)

Endothelial dysfunction without coronary obstruction (e.g.: cocaine use)

\section{2) Damage not related to myocardial ischemia}

Heart bruising

Surgical incision

Radiofrequency or ablation procedure

Rhabdomyolysis with cardiac involvement

Myocarditis

Cardiotoxic agents, such as anthracyclines, herceptin carbon monoxide poisoning

Burns over $30 \%$ of body surface

\section{3) Multifactorial or indeterminate group}

Apical ballooning syndrome

Severe pulmonary embolism or pulmonary hypertension

Peripartum cardiomyopathy

Acute neurological disease (e.g.: stroke, trauma)

Infiltrative diseases (e.g.: Amyloidosis, sarcoidosis)

Extreme exerrcise

Sepsis

Acute respiratory failure

Multiple defibrillation 
The peak time and duration of troponin response depend on the mechanism of the injury, the dose and the frequency of the administration test. A preanalytical evaluation of the method used in each institution must be made by its medical staff. It is recommended by consensus that the value of troponin represented by myocardial injury is the value above the 99th percentile of that in healthy people and that the coefficient of variation is $<10 \%$.

Because the overvaluation of the troponin in some guidelines related to heart disease, several clinicians interpret the increased level of troponin as a synonym of $\mathrm{AMI}$, resulting in an undervaluation of the clinical signs of myocardial ischemia. This must have led to an over diagnosis of AMI and the negative consequences was that its treatment may cause bleeding and complications of coronary angiography without the benefit of the diagnosis of AMI, when these measures are adopted. Thus, the interpretation of a positive troponin should be linked to the more classic symptoms of each disease and associated with other predictors that can improve the diagnosis, as it was showed in the study of Alcalai et al, ${ }^{(24)}$ in which among 615 patients with elevated troponin $\mathrm{T} ; 53 \%$ had a diagnosis of acute coronary syndrome, in the remaining $41 \%$ the increase was due to nonthrombotic troponin elevation and in $6 \%$ the cause could not be identified.

The first generation of the cTn allowed detection in 4-12 hours after the onset of the myocardial injury peaking between 12 to 48 hours. This marker has an area under the curve - receiver operating characteristic curve (ROC) - similar to CK-MB. The ROC curve is a graph that expresses the sensitivity versus 1 -specificity and it is used to evaluate the performance of a test. The new tests of cTn high sensitivity (cTn- hs) provides more than $90 \%$ of sensitivity for the diagnosis of AMI within 2 hours of symptoms. With the possibility of detection of troponin on tenfold lower values and increased sensitivity in the first 2-3 hours of admission, the ability to exclude or diagnose AMI was increased and the possibility of early discharge was greater. ${ }^{(25)}$

The main objective of further testing of cTn-hs is to hasten the diagnosis and to implement therapy earlier, reducing the overcrowding of emergency rooms. But the implications of early diagnosis need to be evaluated carefully. Regardless of whether the test is or is not sensitive enough, the positivity of this marker needs to be contextualized regarding the clinical conditions and the necessary causes of its increase should be recognized in order to avoid the over diagnosis of an acute coronary syndrome.

It is recommended that, in the presence of chest pain, if the first cTn-hs is normal, the second sample is measured in three hours, and if there is an increase $\geq 50 \%$, the diagnosis of AMI can be confirmed (increase $\geq 20 \%$ also was able to establish this diagnosis). Thus, minor changes in troponin, below $20 \%$ of the normal value, are considered sufficient to rule out AMI when the clinical situation is clear and the pretest probability is low for ACS. ${ }^{(26)}$

The kinetics of troponin is essential when trying to differentiate chronic from acute myocardial damage, as well as if the appropriate increase is, or is not, secondary to myocardial ischemia (AMI type 1). The higher the value, the greater the likelihood of AMI. ${ }^{(27)}$. However, there is no clear numerical definition for this distinction between two diseases that can occur independently or in an associated manner. Considering APE with positive myocardial necrosis marker, either elevated CK-MB or troponin, as a clinical expression of ACS, can generate greater cost and risk, both by the higher request of cardiac catheterization, as well as with the longer hospital stays.

Therefore, AMI definition is subject to confounders, by the lack of specificity of the clinical condition, changes in the electrocardiogram and increases of troponin. Since dyspnea can be a presentation of myocardial ischemia, as evidenced in the GRACE registry(28) and Thygesen et al( $^{(26)}$ and also recognizing that chest pain can be an expression of other acute cardiac diseases, the definition of APE precipitated by an unstable atherosclerotic plaque is virtually impossible, except in STEMI, where the ECG has a characteristic evolution.

Rising of troponin in acute or chronic HF has no clear mechanism; it has been attributed to the myocardial injury, the myocardial infarction type 2 or the use of drugs to treating acute decompensated HF. Differentiating whether the increased troponin 
caused or was caused by the ADHF is a major challenge for the clinician. Some authors suggest coronary angiography for this differentiation, which is difficult, since the main cause of heart failure is coronary artery disease itself. Moreover, the only certainty that increased troponin would be caused by an acute coronary event would be the angiographic visualization of intracoronary thrombus associated with a correlation between electrocardiographic findings and the culprit artery.

Most studies that have defined percutaneous intervention as the best treatment in the presence of an ACS had as inclusion criteria the symptom of chest pain suggestive of myocardial ischemia in the last 24 hours of admission, associated with increased markers of myocardial necrosis level and/ or ECG changes compatible with ischemia. In these studies, there is no referral of inclusion of APE as clinical criteria. So to consider patients with APE and elevated level of marker of myocardial necrosis as an ACS and treat them with antiplatelet drugs and percutaneous intervention is a course of action that still needs confirmation in studies.

The likelihood of misdiagnosis increases when electrocardiographic changes are observed, as marked T wave inversion and increased QT interval, in the presence of the EAP, without representing an acute coronary. ${ }^{(29)}$

Thus, the challenge remains, and further studies are needed to aid in accurate diagnosis of both conditions (APE and ACS) as well as cases in which one results from another, because the role of coronary artery disease in acute heart failure has not been properly studied and understood in clinical trials. (9) This question will probably be solved if a new test of high sensitivity and specificity, not only to detect myocardial injury, but to detect necrotic injury due to ischemia, should be developed. In addition, acute pulmonary edema should also be included in the next randomized clinical trials of ACS without elevation of ST.

\section{REFERENCES}

1. Ware LB, Matthay MA. Acute Pulmonary Edema. N Engl J Med. 2005;353(26):2788-96.

2. Steg PG, Dabbous OH, Feldman LJ, CohenSolal A, Aumont MC, Lopez-Send J, et al. Determinants and Prognostic Impact of Heart Failure Complicating Acute Coronary Syndromes: Observations from the Global Registry of Acute Coronary Events (GRACE). Circulation. 2004;109(4):494-9.

3. Montera MW, Almeida RA, Tinoco EM, Rocha RM, Moura LAZ, Réa-Neto A, et al. Sociedade Brasileira de Cardiologia. II Diretriz Brasileira de Insuficiência Cardíaca Aguda. Arq Bras Cardiol. 2009;93(3 supl.3):1-65.

4. Weintraub NL, Collins SP, Pang PS, Levy PD, Anderson AS, Arslanian-Engoren C, et al. Acute heart failure syndromes: Emergency department presentation, treatment, and disposition: Current approaches and future aims: A scientific statement from the American Heart Association. Circulation. 2O10;122(19):1975-96.

5. Adams KF, Fonarow GC, Emerman CL, Le Jemtel TH, Costanzo MR, Abraham WT, et al. Characteristics and outcomes of patients hospitalized for heart failure in the United States: Rationale, design, and preliminary observations from the first 100,000 cases in the Acute Decompensated Heart Failure National Registry (ADHERE). Am Heart J. 2005;149(2):209-16.

6. Harjola VP, Costa S, Sund R, Ylikangas S, SiiriläWaris K, Melin J, et al. The type of acute heart failure and the costs of hospitalization. Int J Cardiol. 2010;145(1):103-5.

7. Pena-Gil C, Figueras J, Soler-Soler J. Acute cardiogenic pulmonary edema. Relevance of multivessel disease, conduction abnormalities and silent ischemia. Int J Cardiol. 2005;103(1):59-66.

8. Fox KF, Cowie MR, Wood DA, Coats AJ, Gibbs JS, Underwood SR, et al. Coronary artery disease as the cause of incident heart failure in the population. Eur Heart J. 2001;22(3):228-36. 
9. McMurray JJ, Adamopoulos S, Anker SD, Auricchio A, Böhm M, Dickstein K, et al. ESC Guidelines for the diagnosis and treatment of acute and chronic heart failure 2012: The Task Force for the Diagnosis and Treatment of Acute and Chronic Heart Failure $2 \mathrm{Ol} 2$ of the European Society of Cardiology. Developed in collaboration with the Heart Failure Association (HFA) of the ESC. Eur J Heart Fail. 2012;14(8):803-69.

1O. Messerli FH, Bangalore S, Makani H, Rimoldi SF, Allemann Y, White CJ, et al. Flash pulmonary oedema and bilateral renal artery stenosis: The Pickering Syndrome. Eur Heart J. 2011;32(18):2231-5.

11. Collins SP, Lindsell CJ, Storrow AB, Abraham WT. Prevalence of negative chest radiography results in the emergency department patient with decompensated heart failure. Ann Emerg Med. 2006;47(1):13-18.

12. Yancy CW, Jessup M, Bozkurt B, Butler J, Casey DE Jr, Drazner MH, et al. 2013 ACCF/AHA Guideline for the Management of Heart Failure: A Report of the American College of Cardiology Foundation/American Heart Association Task Force on Practice Guidelines. Circulation. 2013;128(16):e24O-327.

13. Januzzi JL Jr, Camargo CA, Anwaruddin S, Baggish AL, Chen AA, Krauser DG, et al. The $\mathrm{N}$-terminal Pro-BNP investigation of dyspnea in the emergency department (PRIDE) study. Am J Cardiol. 2005;95(8):948-54.

14. Wang CS, FitzGerald JM, Schulzer M, Mak E, Ayas NT. Does this dyspneic patient in the emergency department have congestive heart failure? JAMA. 2005;294(15):1944-56.

15. Biagi RW, Bapat BN. Frusemide in acute pulmonary oedema. Lancet. 1967;1(7494):849.

16. Owen DR, MacAllister R, Sofat R. Intravenous Furosemide for Acute Decompensated Congestive Heart Failure: What Is the Evidence? Clin Pharmacol Ther. 2015;98(2):119-21.

17. Weng $C L$, Zhao YT, Liu $Q H$, Fu CJ, Sun F, Ma YL, Chen YW, et al. Meta-analysis : Noninvasive Ventilation in Acute Cardiogenic Pulmonary. Ann Int Med. 2010;152(9):590-600.
18. Javed U, Aftab W, Ambrose JA, Wessel RJ, Mouanoutoua M, Huang G, et al. Frequency of Elevated Troponin I and Diagnosis of Acute Myocardial Infarction. Am J Cardiol. 2009;104(1):9-13.

19. Figueras J, Peña C, Soler-Soler J. Thirty day prognosis of patients with acute pulmonary oedema complicating acute coronary syndromes. Heart. 2005;91(7):889-93.

2O. Piegas LS, Avezum A, Guimarães HP, Muniz AJ, Reis HJ, Santos ES, et al. Acute coronary syndrome behavior: results of a Brazilian registry. Arq Bras Cardiol. 2013;10O(6):502-10.

21. Katus HA, Remppis A, Neumann FJ, Scheffold T, Diederich KW, Vinar G, et al. Diagnostic efficiency of troponin T measurements in acute myocardial infarction. Circulation. 1991;83(3):902-12.

22. Alpert JS, Thygesen K, Antman E, Bassand JP. Myocardial Infarction Redefined - A Consensus Document of The Joint European Society of Cardiology / American College of Cardiology Committee for the Redefinition of Myocardial Infarction: The Joint European Society of Cardiology/ American College of Cardiology Committee. J Am Coll Cardiol. 2000;36(3):959-69.

23. Thygesen K, Mair J, Katus H, Plebani M, Venge $\mathrm{P}$, Collinson P, et al. Recommendations for the use of cardiac troponin measurement in acute cardiac care. Eur Heart J. 2010;31(18):2197-206.

24. Alcalai R, Planer D, Culhaoglu A, Osman A, Pollak A, Lotan C. Acute coronary syndrome vs nonspecific troponin elevation: clinical predictors and survival analysis. Arch Intern Med. 2007;167(3):276-81.

25. Reichlin T, Hochholzer W, Bassetti S, Stever S, Stelzig C, Hartwiger S, et al. Early Diagnosis of Myocardial Infarction with Sensitive Cardiac Troponin Assays. N Engl J Med. 2009;361(8):858-67.

26. Thygesen K, Mair J, Giannitsis E, Mueller C, Lindahl B, Blankenberg $\mathrm{S}$, et al. How to use highsensitivity cardiac troponins in acute cardiac care. Eur Heart J. 2012;33(18):2252-57.

27. Thygesen K, Alpert JS, Jaffe AS, Simoons ML, Chaitman BR, White HD, et al. Third universal definition of myocardial infarction. Eur Heart J. 2012;33(20):2551-67. 
28. Thygesen K, Alpert JS, White HD, Jaffe AS, Apple FS, Galvani M, et al. Universal definition of myocardial infarction. Eur Heart J. 2007;28(20):2525-38.
29. Littmann L. Large T wave inversion and QT prolongation associated with pulmonary edema: a report of nine cases. J Am Coll Cardiol. 1999;34(4):1106-10. 